اثر سطوح مختلف ويتامين C و عنصر روى بر خصوصيات كيفى اسيرم قوج عربى

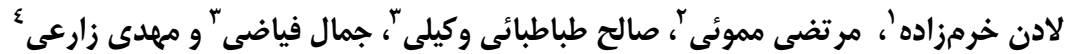

ا- دانش أموخته كارشناسى ارشد فيزيولوزى دام دانشكاه علوم كشاورزى و منابع طبيعى خوزستان

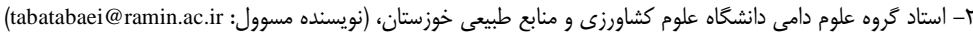

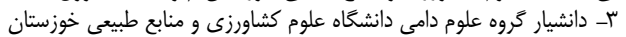

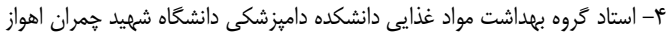

تاريخ بذيرش:

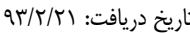

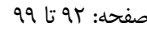

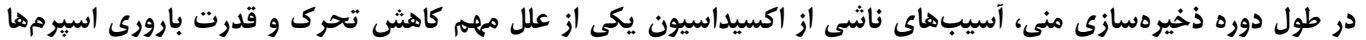

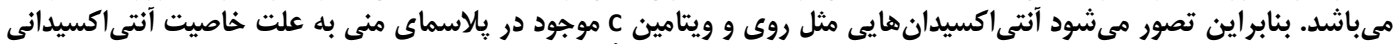

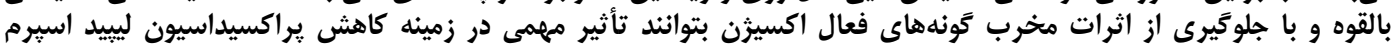

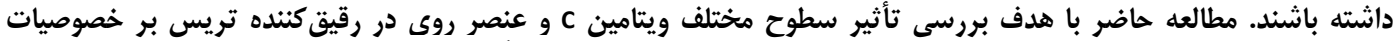

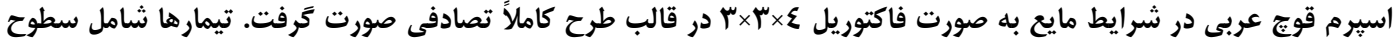

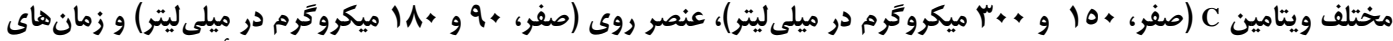

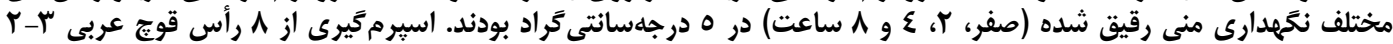

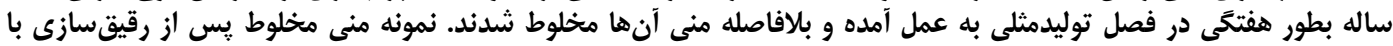

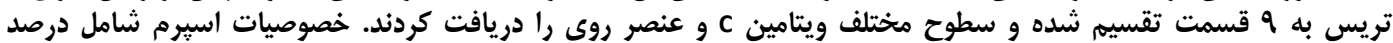

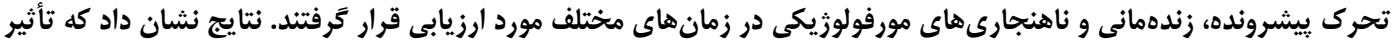

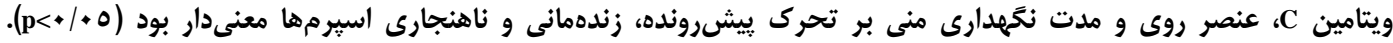

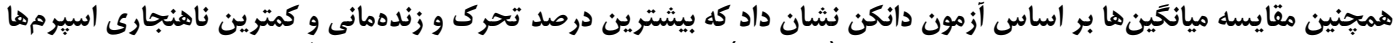

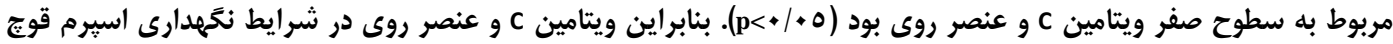

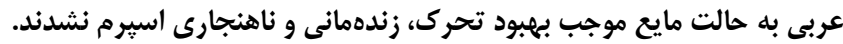

وازههاى كليدى: روى، قوج عربى، كيفيت منى، ويتامين C

تركيبات به اين صورت است كه با دادن يك اتم هيدروثن به زيه

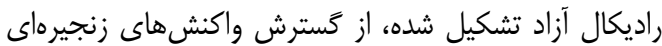

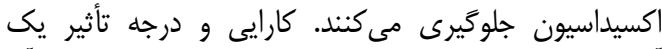

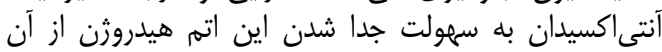

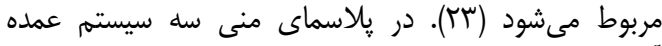

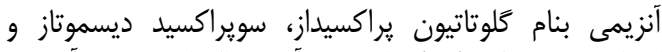

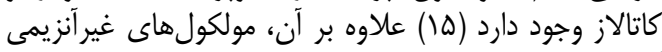

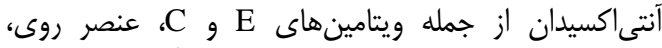

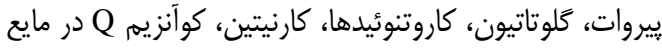

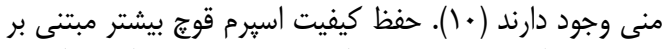

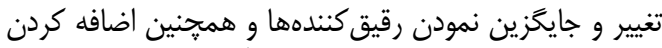

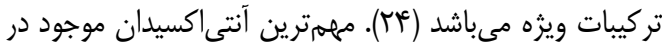

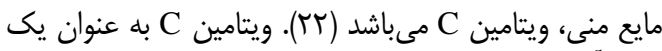

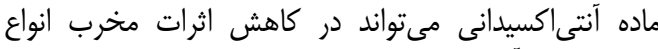

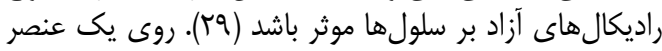

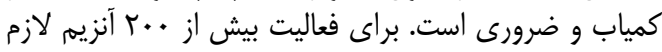

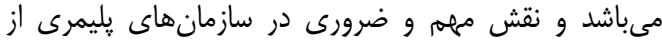

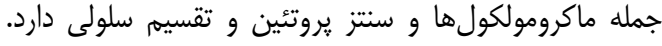
روى نقش مهمى در عملكرد يروستات، بيضه و و إيبديديم داردم.

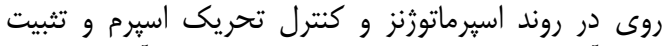

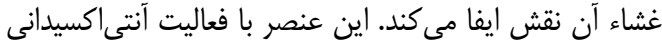

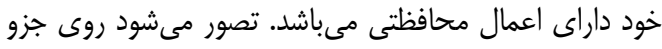

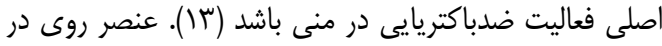

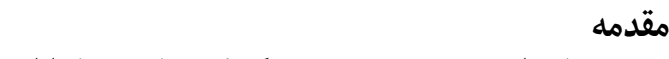

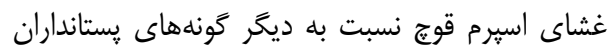

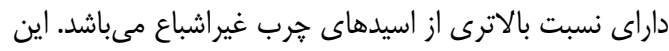

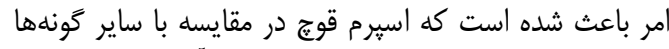

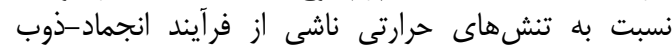

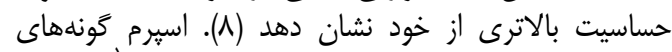

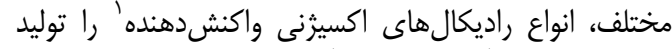

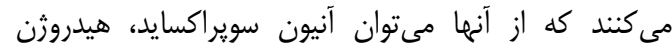

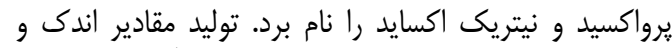

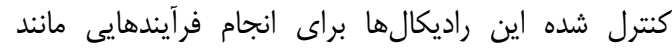

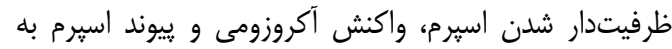

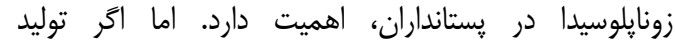

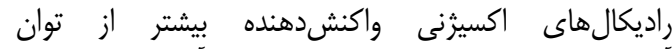

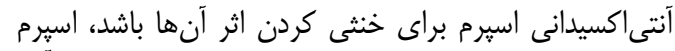

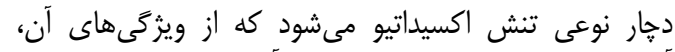

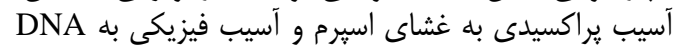

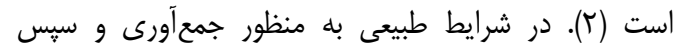

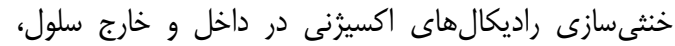

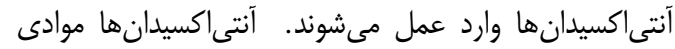

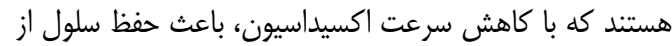

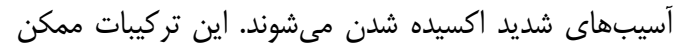

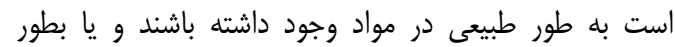

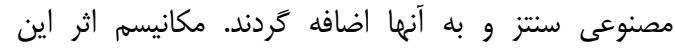


فراسنجههاى كيفى اسيرم قرار گرفتند. به منظور تعيين درصد

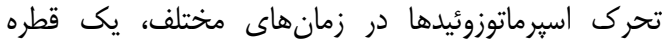

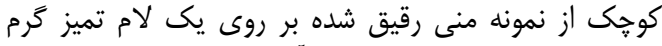

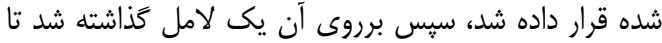

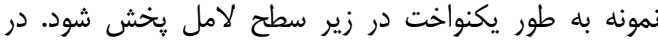

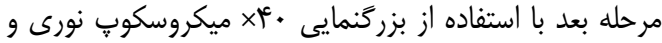

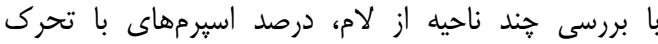

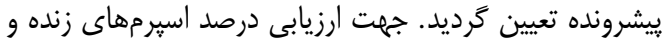

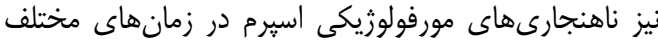

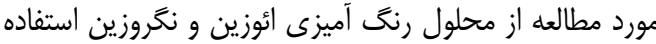

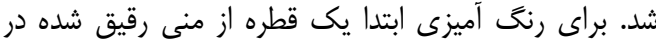

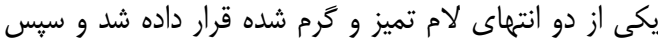

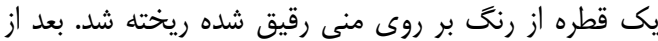

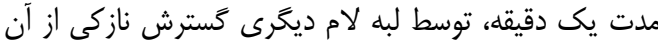

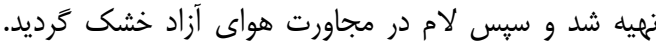

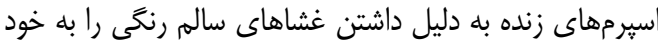

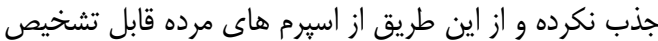

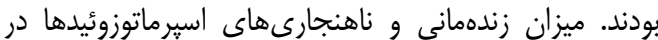

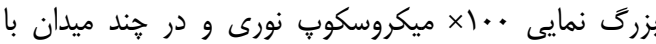

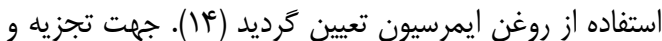

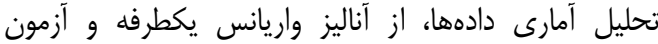

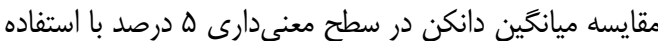
از نرمافزار SAS نسخه / /9 استفاده شد.

\section{نتايج و بحث}

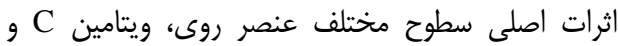

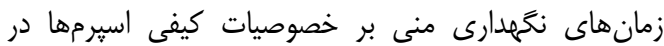

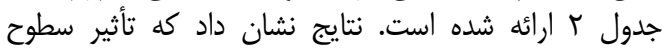

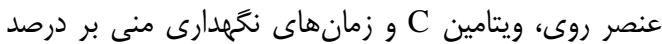

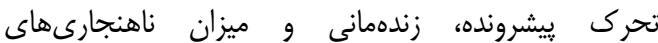

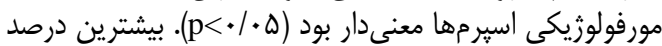

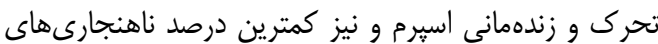

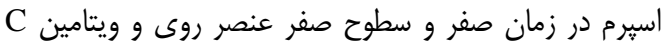

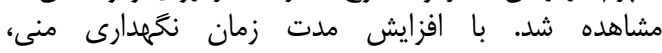

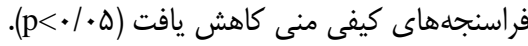

تثبيت غشاى یاساسمايى اسيرماتوزوئيدها نقش فعالى دارد

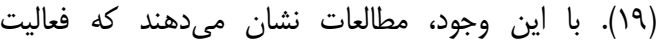

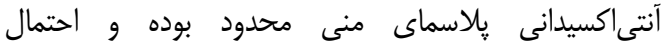

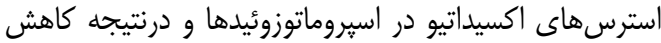

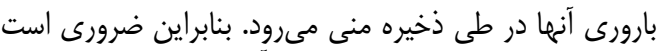

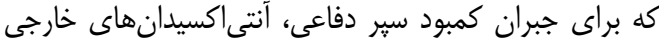

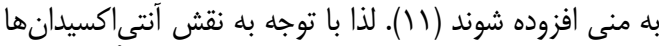

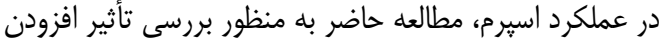

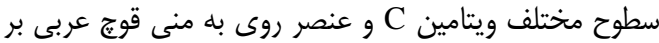

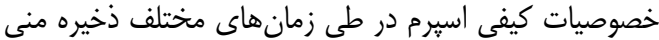

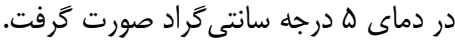

\section{مواد و روشها}

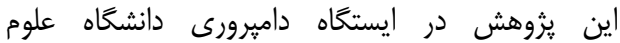

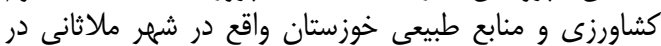

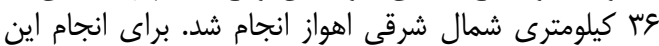

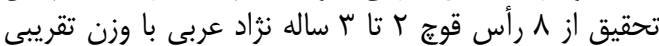

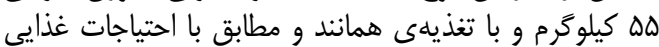

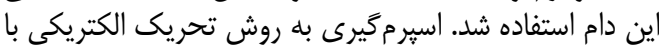

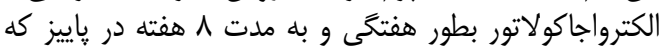

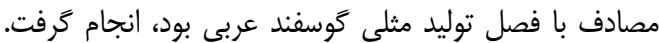

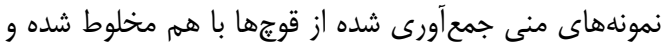

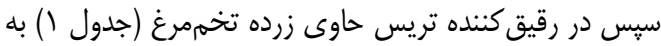

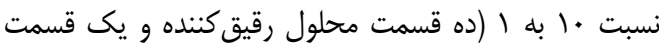

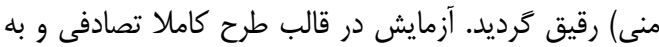

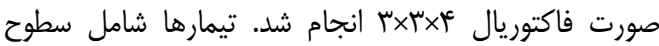

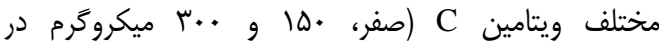

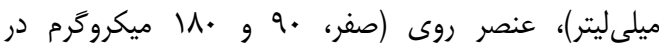

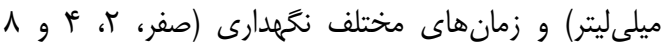

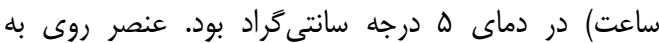

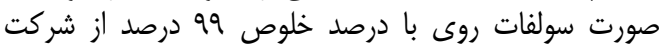

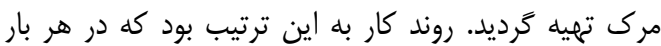

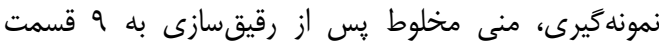

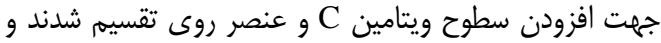
در زمانهاى مختلف نكمهدارى در دماى فوق مورن مورد ارزيابى 
جدول ا- فرمول رقيق كننده تريس حاوى زرده تخم مرغ جهت نكحهدارى منى قوج عربى در شرايط مايع (سالامون و ماكسول، ...") Table 1. Tris extender formula containing egg yolk to protect the ram semen under liquid condition

\begin{tabular}{|c|c|}
\hline 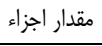 & اجزاى رقيق كننده \\
\hline$r / 9 \mu \mathrm{F}$ & تريس (هيدروكسى متيل آمينومتان) (كرم) \\
\hline$\cdot 10$ & فروكتوز (كرم) \\
\hline 1/99 & اسيد سيتريك (منوهيدرات) (كرم) \\
\hline 14 & زرده تخهمرغ (ميلىليتر) \\
\hline $1 .$. & آب مقطر (ميلىليتر) \\
\hline
\end{tabular}

جدول r- مقايسه ميانگين اثرات اصلى سطوح مختلف روى، ويتامين C و مدت نكَهدارى منى بر فراسنجهاى كيفى اسبرمها (درصد) در قوج عربى Table 2. Comparison the main effects of different levels of zinc, vitamin $\mathrm{C}$ and semen storage period on sperm quality parameters (\%) in Arabi ram

\begin{tabular}{|c|c|c|c|c|}
\hline ن اهنجارى & ز زنده مانى & تحرى ييشرونده & & \\
\hline$g / V \Lambda^{C} \pm \cdot / T V$ & $V W / r^{a^{a} \pm \cdot / V \Delta}$ & $99 / 1 .^{a} \pm \cdot / v 9$ & صفر & \\
\hline$q / 4 v^{b} \pm \cdot / r^{\prime} q$ & $V \Delta / r \cdot{ }^{b} \pm \cdot / \Lambda V$ & $\Delta V / \Gamma \mu^{b} \pm 1 / \mu^{\prime}$ & 9. & روى \\
\hline $11 / r^{a} \pm \cdot / 4 r$ & $\checkmark \backslash / \mathcal{F}^{c} \pm \cdot / q$. & $\Delta \varepsilon / \cdot \wedge^{b} \pm 1 / r v$ & $1 \wedge$. & (ميكرو گرم در ميلىليتر) \\
\hline$V / \cdot r^{C} \pm \cdot / T V$ & $\Lambda \cdot / .^{a} \pm \cdot / v r$ & $V T / / F^{a} \pm \cdot / \Lambda$. & صفر & \\
\hline $1 . / N \varepsilon^{a} \pm \cdot / \& \Delta$ & $V T / q^{b} \pm \cdot / 19$ & $\Delta r / / \varphi^{c} \pm 1 / \Gamma$. & 10 . & C ويتامين C \\
\hline$\left.q / 99^{b} \pm . / 4\right)$ & $V / / \Lambda \cdot{ }^{b} \pm \cdot / v e$ & $\Delta V / \Lambda)^{b} \pm 1 / 11$ & $r .$. & (ميكرو كرم در ميلى ليتر) \\
\hline$\varepsilon\left|\wedge r^{d} \pm \cdot / r\right|$ & $\Lambda T / F \cdot{ }^{a} \pm \cdot / g V$ & $q N / T^{a} \pm I / R^{2} q$ & صفر & \\
\hline$\Lambda / \digamma^{c} \pm \cdot /{ }^{c} \Lambda$ & $V v / r v^{b} \pm \cdot / q q$ & $q \pi / r \cdot{ }^{b} \pm 1 / 4 q$ & r & زمان \\
\hline$q / \wedge v^{b} \pm \cdot / \wedge \gamma^{c}$ & $V T / r \cdot{ }^{c} \pm \cdot / W V$ & $\Delta q / T F^{C} \pm 1 / \kappa r$ & r & (ساعت) \\
\hline $11 / \wedge 9^{a} \pm \cdot / \Delta \Delta$ & $s \varepsilon / 9 V^{d} \pm \cdot / \lambda f^{2}$ & $\Delta r / F q^{d} \pm 1 / \kappa F^{c}$ & $\wedge$ & \\
\hline
\end{tabular}




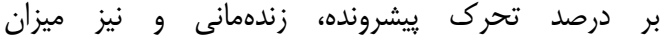

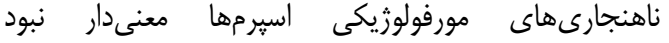
$\cdot(\mathrm{p}>\cdot 1 \cdot \Delta)$
مقايسه ميانگين اثرات متقابل سطوح مختلف عنصر روى،

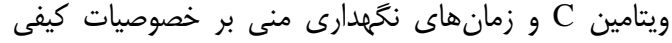

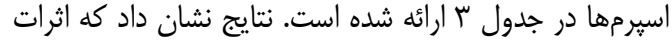

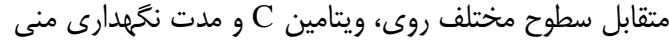

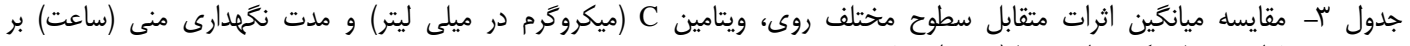

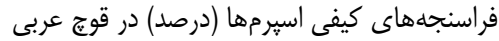

Table 3. Comparison the interaction effects of different levels of zinc, vitamin $C(\mu \mathrm{g} / \mathrm{ml})$ and semen storage period (h) on sperm quality parameters (\%) in Arabi ram

\begin{tabular}{|c|c|c|c|c|c|}
\hline ناهنجارى & زنده مانى & تحرى & زمان & ويتامين C & روى \\
\hline$r / r V \pm \cdot / F r$ & $\Lambda \varepsilon / \Gamma \Delta \pm 1 / \Gamma V$ & $\Lambda \cdot / r \Delta \pm r / \Lambda \Delta$ & صفر & صفر & \\
\hline$\Delta / \Gamma \Delta \pm \cdot / F \Delta$ & $\Lambda r / r V \pm I / \Delta)$ & $V r / G T \pm r / V r$ & r & & \\
\hline G/GTE./VA & $V \varepsilon / V \Delta \pm r / r$. & $s q / \Delta \cdot \pm r / r \varphi$ & f & & \\
\hline $9 / 8 T \pm 1 / 91$ & $V \cdot / r V \pm r / \cdot r$ & $q r / r \Delta \pm r / r q$ & $\wedge$ & & \\
\hline$\Delta / T V \pm \cdot / F T$ & $\Lambda \Delta / r V \pm \cdot / K r$ & $v \Delta / v \Delta \pm 1 / 9 \Delta$ & صفر & 10. & صفر \\
\hline$g / T r \pm \cdot / r r$ & $V N / V \Delta \pm V / \Delta \Delta$ & $V / / \cdot \pm r / \cdot V$ & r & & \\
\hline$\Lambda / r \Delta \pm \cdot / \Lambda r$ & $V E / V \Delta \pm 1 / F)$ & $\varepsilon g / r \Delta \pm r / \Lambda \Lambda$ & f & & \\
\hline $9 / 9 Y \pm \cdot / Q 1$ & $G N / \mid r \pm I / W V$ & $G \cdot / G T \pm T / 1 D$ & $\wedge$ & & \\
\hline$\Delta / / r \pm \cdot / r q$ & $\Lambda 1 / \Delta \cdot \pm 1 / \cdot \Lambda$ & $V Q / I T \pm I / r r$ & صفر & r.. & \\
\hline$\Delta / \& Y \pm \cdot / T r$ & $V N / V \Delta \pm I / \backslash \varphi$ & $\checkmark / \vee \Delta \Delta \pm \cdot / \wedge \varepsilon$ & r & & \\
\hline$\varepsilon / V \Delta \pm \cdot / \Psi \varepsilon$ & $V \pi / \Delta \cdot \pm 1 / ৭ \varphi$ & $G N / T V \pm \cdot / A r$ & f & & \\
\hline$N / r V \pm \cdot / F r$ & $V \cdot / r \Delta \pm I / \tau F$ & $q \pi / / r \pm 1 / \cdot q$ & $\wedge$ & & \\
\hline$\Delta / \Delta \cdot \pm \cdot / T V$ & $\Lambda V / T V \pm \cdot / \Delta q$ & $\Lambda 1 / \Delta \cdot \pm r / r \varepsilon$ & صفر & صفر & \\
\hline$s / r v \pm . / M \Lambda$ & $\wedge r / . . \pm 1 / v r$ & $v \varepsilon / . . \pm r / r)$ & r & & \\
\hline$V / T V \pm \cdot / F r$ & $V q / \mid r \pm 1 / \Delta r$ & $\checkmark / r Y \pm 1 / \wedge q$ & f & & \\
\hline$N / T \pm \cdot / \Delta \Delta$ & $v \varepsilon / r \Delta \pm \cdot / ৭ q$ & $\left.\varepsilon_{\Delta} / \cdots \pm 1 / \lambda\right)$ & $\wedge$ & & \\
\hline$N / r V \pm I / \cdot V$ & $\Lambda \Gamma / \Delta \cdot \pm I / r \digamma$ & $\Delta \Gamma / v \Delta \pm \cdot / q$. & صفر & 10. & $q$. \\
\hline$q / \vee \Delta \pm / / ৭ \&$ & $V \varepsilon / V \Delta \pm \cdot / q r$ & $\vee q / \wedge V \pm \cdot / \Delta \Delta$ & r & & \\
\hline$|r / r \Delta \pm I / \Delta|$ & $V \backslash / A V \pm I / G r$ & $\forall \Delta / \cdot \cdot \pm \cdot / \wedge \gamma$ & r & & \\
\hline $\mid w / \Delta \cdot \pm 1 / W$ & $q \pi / \Delta \cdot \pm 1 / 4 \pi$ & $r / / V \Delta \pm \cdot / \Lambda$. & $\wedge$ & & \\
\hline$V / I Y \pm \cdot / \Delta D$ & $V q / / r \pm \cdot / V F$ & $\Delta V / r \Delta \pm\rangle / / r$ & صفر & $\mu .$. & \\
\hline $9 / \cdot . \pm \cdot / 91$ & $V r / A V \pm I / A T$ & $\Delta \Gamma / r V \pm 1 / \cdot V$ & r & & \\
\hline$W / r \Delta \pm \cdot / V$. & $98 / 9 r \pm 1 / M$ & $0 \cdot / T V \pm 1 / T{ }^{\epsilon}$ & f & & \\
\hline $\mid Q / \cdot \cdot \pm I / r T$ & qI/rVII/q & $\tau \Psi / \Delta \cdot \pm r / r V$ & $\wedge$ & & \\
\hline$\Delta / V \Delta \pm \cdot / \Delta r$ & $\mathrm{MN} \cdot \cdot \pm \pm \cdot / \mathrm{AT}$ & $V N / \Delta \cdot \pm 1 / \cdot \Delta$ & صفر & صفر & \\
\hline V/GYI./VA & $\Lambda r / / r \pm I / r q$ & $v r / / r \pm \cdot / v q$ & r & & \\
\hline$N / \Delta \cdot \pm \cdot / V \Delta$ & $\vee \vee / \wedge \vee \pm 1 / \cdot \Lambda$ & $s q / v \Delta \pm \cdot / \leftarrow \Delta$ & r & & \\
\hline$q / / r \pm \cdot / \wedge q$ & $V \backslash / \Delta \cdot \pm I / r V$ & $\varangle \leftarrow / \Lambda V \pm \cdot / \uparrow \Lambda$ & $\wedge$ & & \\
\hline 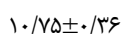 & $r / \Delta \cdot \pm r / \Delta r$ & $\Delta) / g r \pm 1 / r q$ & صفر & 10. & $M$. \\
\hline Ir//rE./Aq & $\varepsilon q / \Lambda V \pm 1 / r)$ & $r q / T Y \pm \cdot / \Delta q$ & r & & \\
\hline $\mid Q / \& T \pm I / \cdot \Lambda$ & $G \Delta / D \cdot \pm I / T r$ & $r \mid / V \Delta \pm \cdot / \Lambda T$ & r & & \\
\hline $\mid V / R V \pm \cdot / \Lambda F$ & $\Delta V / T V \pm I / T)$ & $r V / / r \pm \cdot / \wedge q$ & $\wedge$ & & \\
\hline$q / \cdot \pm \pm 1 / 1 r$ & $\mathrm{~V} / / \cdot \pm \pm \cdot / \Lambda$. & $\Delta q / r V \pm r / 1 q$ & صفر & $r .$. & \\
\hline $1 . / / r \pm 1 / T F$ & $V V / A V \pm . / Q V$ & $\Delta F / G T \pm r / \Delta)$ & r & & \\
\hline $\mid r / T \Delta \pm \cdot / Q T$ & $\varepsilon \Delta / V \Delta \pm 1 / \cdot \varphi$ & $\Delta \cdot / v \Delta \pm r / \cdot \Lambda$ & i & & \\
\hline $\mid g / T \Delta \pm 1 / l f$ & $q \mu / \cdot \pm \cdot / v r$ & $r \Delta / / r \pm r / V V$ & $\wedge$ & & \\
\hline
\end{tabular}


آنتى اكسيدانى محلول در آب است، با اين حال مكمل نمودن

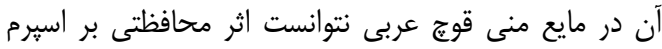

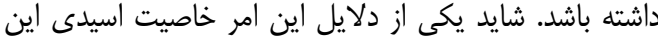

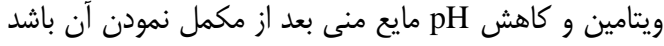

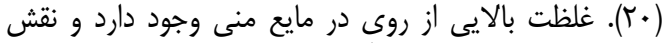

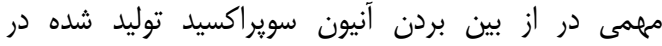

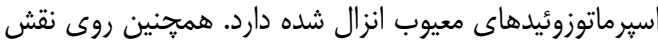

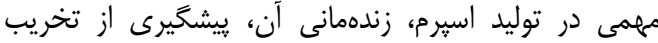

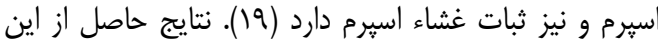

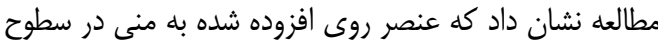

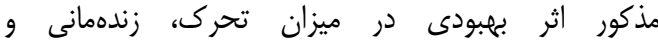

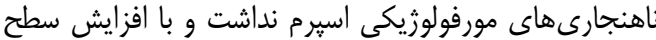

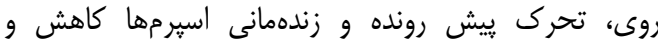

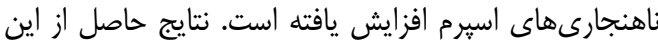

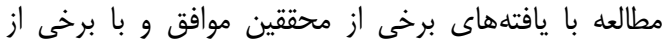

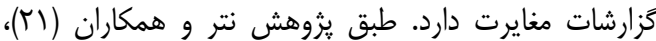

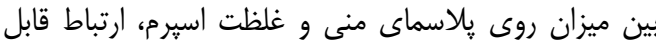

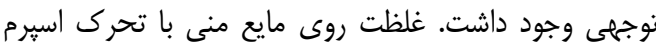

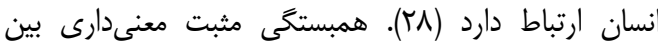

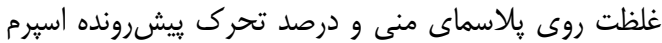

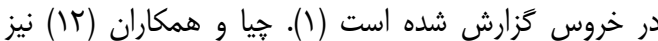

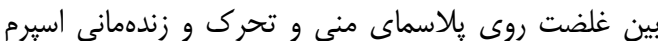

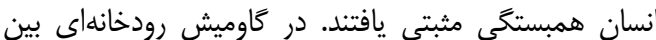

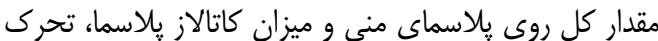

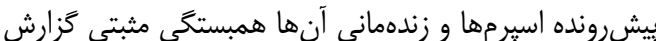

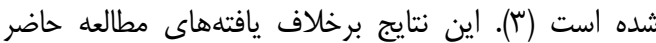

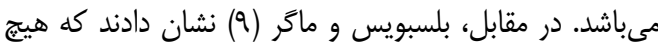

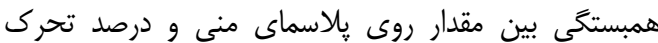

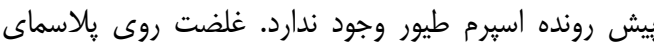

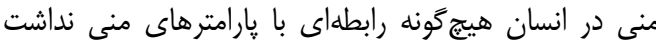

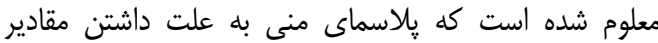

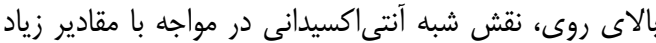

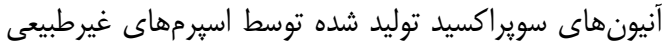

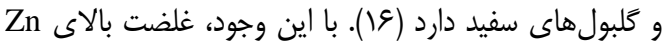

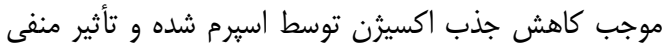

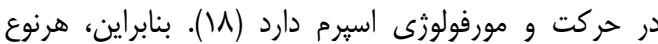

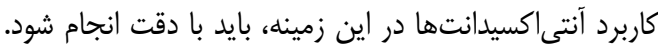

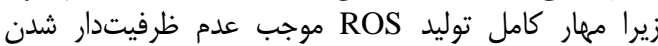

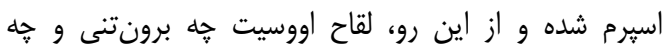

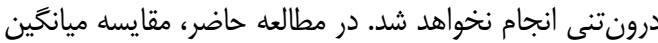

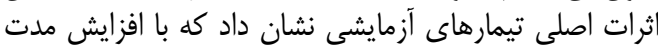

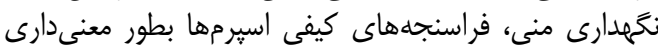

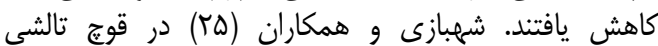

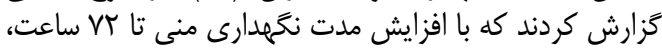

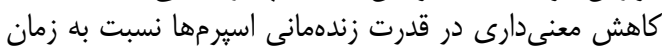

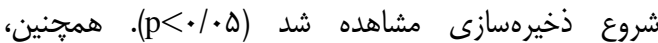

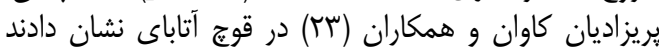

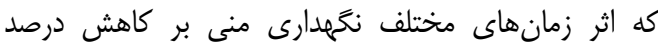
اسيرمهاى متحرك و زنده در شرايط نمائ مايع معنىدار بودند.
غشاى اسيرم به علت دارا بودن مقادير بالايى از اسيدهاى

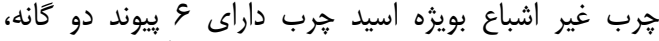

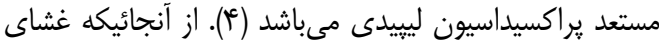

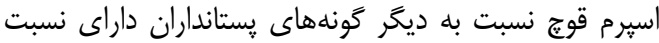

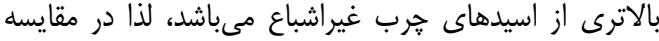

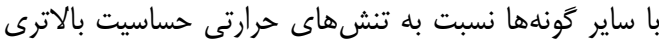

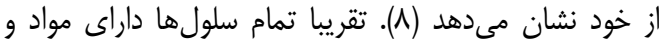

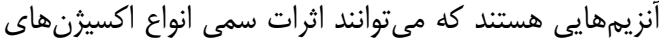

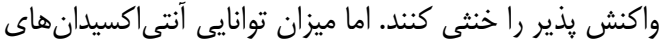

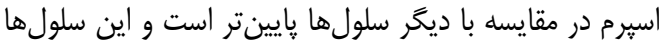

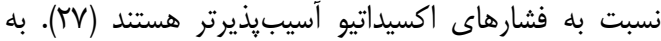

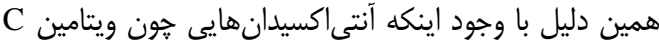

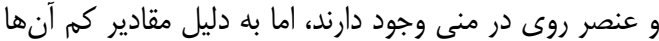

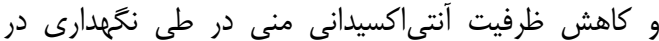

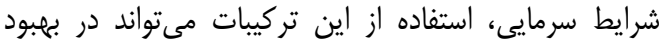

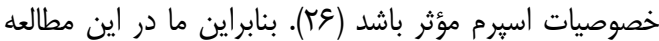

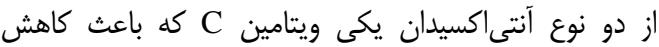

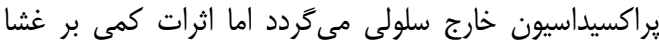

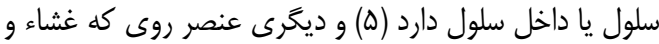

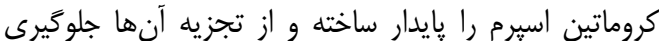

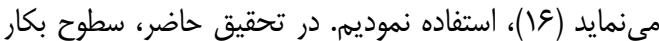

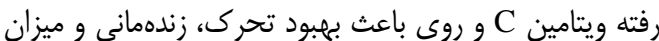

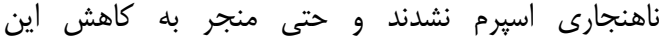

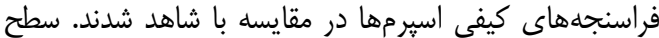

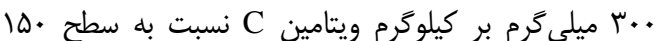

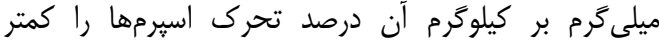

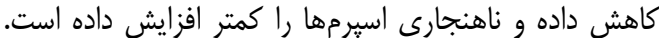

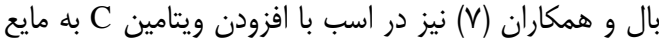

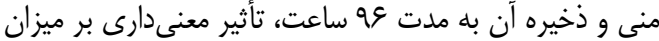

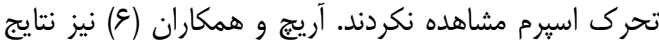

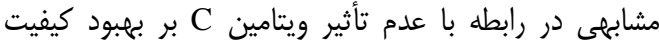

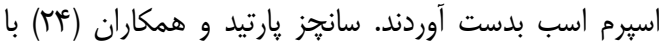

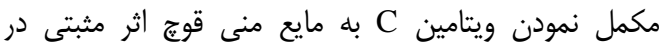

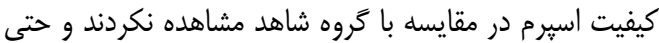

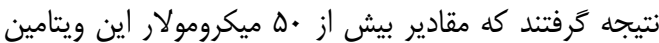

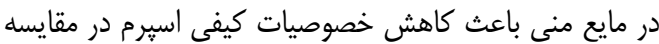

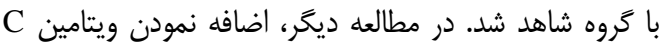

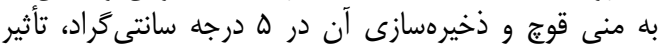

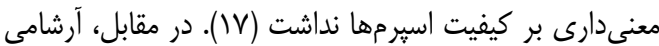

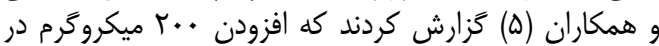

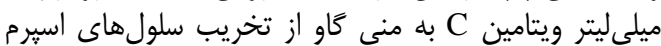

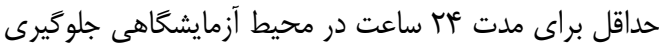

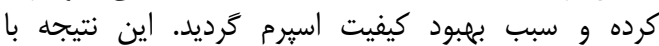

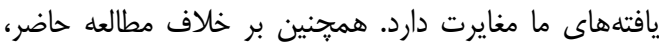

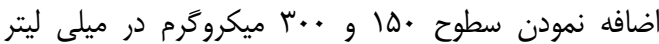

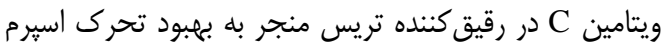

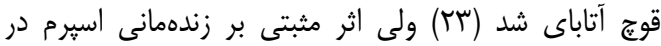

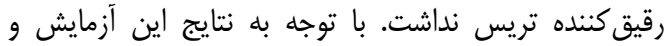

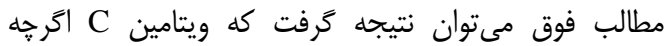




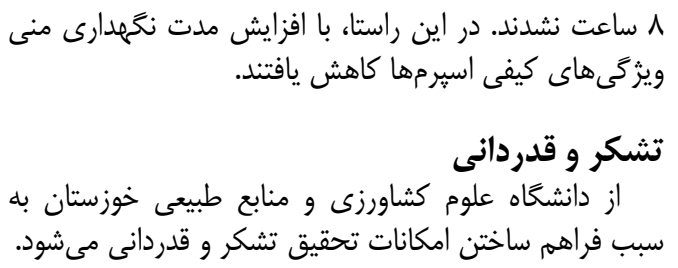

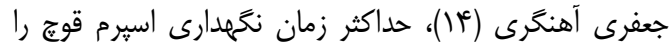

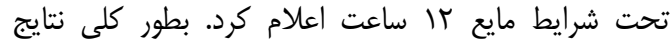

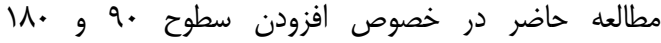

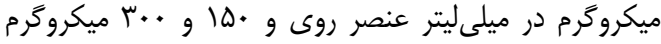

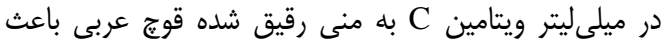

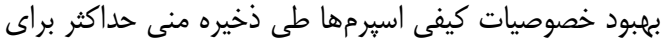

1. Aghaei, A., S. Tabatabaei and M. Nazari. 2010. The correlation between mineral concetration of seminal plasma and spermatozoa motility in rooster. Journal of Animal and Veterinary Advances, 9(10): 1476-1478.

2. Aitken, R.J., G.N. de Iuliis, J.M. Finnie, A. Hedges and R.I. McLachlan. 2010. Analysis of the relationships between oxidative stresses, DNA damage and sperm vitality in a patient population: development of diagnostic criteria. Human Reproduction, 25: 2415-2426.

3. Alavi-shoushtari, S.M., M.H. Asri Rezai, Kh. Ansari and A. Khaki. 2009. Effects of the seminal plasma zinc content and catalase activity on the semen quality of water buffalo (Bubalus bubalis) bulls. Pakistan Journal of Biological Sciences, 12(2): 134-139.

4. Alvarez, J.G., J.C. Touchstone, L. Blasco and B.T. Storey. 1987. Spontaneous lipid peroxidation and production of hydrogen peroxide and superoxide in human spermatozoa superoxide dismutase as a major enzyme protectant against oxygen toxicity. Journal of Andrology, 8(5): 338-48.

5. Arshami, J., H. Esmailzadeh, M. Nasiri Mahallati and M. Hashemi Attar. 1385. Evaluation the in vitro effects of vitamin $\mathrm{E}$ and vitamin $\mathrm{C}$ on spermatozoa characteristics. Iranian Journal of Medical Sciences, 9(2): 75-82. (In Persian)

6. Aurich, J.E., U. Schonherr, H. Hoppe and C. Aurich. 1997. Effects of antioxidants on and membrane integrity of chilled stored stallion semen. Theriogenology, 48: 185-192.

7. Ball, B.A., V. Medina, C.G. Gravance and J. Baumber. 2001. Effect of antioxidants on preservation of motility, viability and acrosomal integrity of equine spermatozoa during storage at $5^{\circ} \mathrm{C}$. Theriogenology, 56: 577-589.

8. Baumber, J., B.A. Ball, C.G. Gravance, V. Medina and M. Davies-Morel. 2000. The effect of reactive oxygen species on equine sperm motility, viability, acrosomal integrity, mitochondrial membrane potential, and membrane lipid peroxidation. Journal of Andrology, 21: 895.

9. Blesbois, E. and I. Mauger. 1989. Zinc content of fowl seminal plasma and its effects on spermatozoa after storage at 4 degrees C. British Poultry Science, 30: 677-685.

10. Bonnes Taourel. D., M.C. Guerin and J. Torreilles. 1992. Is malodialdhyde a valuable indicator of lipid peroxidation. Biochemical Pharmacology, 44(5): 985-988.

11. Bruemmer, J.E., R.C. Coy, E.L. Squires and J.K. Graham. 2002. Effect of pyruvate on the function of stallion spermatozoa stored for up to 48 hours. Journal of Animal Science, 80: 250-267.

12. Chia, S.E., C.N. Ong, L.H. Chau, L.M. Ho and S.K. Tay. 2000. Comparison of zinc concentrations in blood and seminal plasma and the various sperm parameters between fertile and infertile men. Journal of Andrology, 21: 53-57.

13. Gavella, M. and V. Lipovac. 1998. In vitro effect of zinc on oxidative changes in human semen. Andrologia, 30: 317-323.

14. Jafari Ahangari, Y. 1996. An investigation on the effect of various buffers (Tris, Citrate and Skimmed milk) on ram semen motility and survival characteristics in liquid storage. Final report of Research Plan. Animal Science Research Institute, $37 \mathrm{pp}$.

15. Kankofer, M., G. Kolm, J. Aurich and C. Aurich. 2005. Activity of glutathione peroxidase, superoxide dismutase and catalase and lipid peroxidation intensity in stallion semen during storage at 5 degree C. Theriogenology. 63: 1354-1365.

16. Kharazi, H., A, Vaisi Raigani, B. Etesami, M. Khazae, A. Kiani, E. Rafiee Alavi and S.S. Shahrokhi. 2011. Comparison of anti-oxidant enzymes activity and levels of zinc and selenium in sperm and seminal plasma between fertile and idiopathic infertile men. Kermanshah Behbood J. 14(4): 316-327 (In Persian).

17. Kheradmand, A., H. Babaei and J. Abshenas. 2006. Comparative evaluation of the effect of antioxidants on the chilled-stored ram semen. Iranian Journal of Veterinary Research, 7: 40-45.

18. Kvist, U., S. Kjellberg and L. Bjorndahl. 1988. The role of zinc in sperm chromatin stability in fertile men. Scandinavian Journal of Urology and Nephrology, 22(1): 1-6.

19. Lewis-Jones, D.I., I.A. Aird, M.M. Biljan and C.R. Kingsland. 1996. Effects of sperm activity on zinc and fructose concentration in seminal plasma. Human Reproduction, 11: 2465-2467. 
20. Mustafa, S. and D. Esref. 2004. The effect of Ascorbic Acid on the freezability of ram semen diluted with extenders containing different proportions of glycerol .Turkish Journal of Veterinary and Animal Sciences, 28: 893-899.

21. Netter, A., R. Hartoma and K. Nahail. 1981. Effects of zinc administration on plasma testosterone and dihydro testosterone and sperm count. Archives of Andrology, 7(1): 69-73.

22. Niki, E. 1991. Action of ascorbic acid as a scavenger of active and stable oxygen radicals American Journal of Clinical Nutrition, 54: 1119-1124.

23. Parizadian Kavan, B., Y. Jafari Ahangari and S. Zerehdaran. 2008. The effects of various levels of Vitamins $\mathrm{E}$ and $\mathrm{C}$ in milk and tris extenders on characteristics of Atabay ram semen in liquid condition. Journal of Agricultural Sciences and Natural Resources, 15(5): 123-131 (In Persian).

24. Sanchez-Partida, L.G., B.P. Setchell and W.M. Maxwell. 1997. Epididymal compounds and antioxidants in. diluents for the frozen storage of ram spermatozoa. Reproduction, Fertility and Development, 9: 689-696.

25. Shahbazi, M., A. Mohit and M. Mohammadi. 2011. Effect of different levels of vitamins E and C on quality of diluted sperm of Taleshi ram during storage at $5^{\circ} \mathrm{C}$. Journal of Veterinary Research, 66(2): 161-164 (In Persian).

26. Sonmez, M. and E. Demirci. 2004. The effect of ascorbic acid on the freezability of ram semen diluted with extenders containing different proportions of glycerol. Turkish Journal of Animal Sciences, 28: 893-899.

27. Sreejith, J.N., A.S. Braar, C.S. Ahuja, S.P.S. Sangha and K.C. Chaudhary. 2006. A comparative study on lipid peroxidation, activities of antioxidant enzymes and viability of cattle and buffalo bull spermatozoa during storage at refrigeration temperature. Animal Reproduction Sciene, 96: 21-29.

28. Stankovic, H. and D. Mikac-Devic. 1976. Zinc and copper in human semen. Clinica chemica Acta, 70(1): 123-126.

29. Yousef, M.I., G.A. Abdollad and K.I. Kamel. 2003. Effect of ascorbic acid and vitamin Esupplementation on semen quality and biochemical parameters of male rabbits. Animal Reproduction Science, 76: 99-111. 


\title{
Effect of Different Levels of Vitamin C and Zinc on Sperm Quality Characteristics of Arabi Ram
}

\author{
Ladan Khoramzadeh ${ }^{1}$, Morteza Mamouei $^{2}$, Saleh Tabatabaei Vakili ${ }^{3}$, Jamal Fayazi $^{3}$ and \\ Mehdi Zarei ${ }^{4}$ \\ 1- Graduated M.Sc. Student of Animal Physiology, Khuzestan Agricultural and Natural Resources University \\ 2- Professor, Department of Animal Science, Khuzestan Agricultural and Natural Resources University \\ 3- Associate Professors, Department of Animal Science, Khuzestan Agricultural and Natural Resources University \\ (Corresponding author: tabatabaei@ ramin.ac.ir) \\ 4- Professor, Department of Food Hygiene, Faculty of Veterinary Medicine, Shahid Chamran Uiversity of Ahwaz \\ Received: May 11, $2014 \quad$ Accepted: July 27, 2016
}

\begin{abstract}
During the semen storage, oxidative damage is one of the major causes of reduced motility and fertility of spermatozoa. Therefore, antioxidants such as zinc and vitamin $\mathrm{C}$ in seminal plasma may be have the important effects on reduce the lipid peroxidation of spermatozoa by prevent the destroy effect of ROS. The aim of this study was to evaluate the effect of different levels of vitamin $\mathrm{C}$ and zinc in tris extender on spermatozoa characteristics of Arabi ram in liquid condition by $3 \times 3 \times 4$ factorial arrangement with the use of completely randomized design. Treatments were included the levels of vitamin C (0,150 and $300 \mathrm{~g} / \mathrm{ml})$, zinc $(0,90$ and 180 $\mathrm{g} / \mathrm{ml})$ and storage periods of diluted semen (0,2, 4 and 8 hours) in refrigerator temperature. Semen was collected weekly from 8 Arabic rams with 2-3 years old in breeding season and mixed together. The mixed semen was divided in to 9 parts and after dilution with tris, vitamin $\mathrm{C}$ and zinc levels were added. Spermatozoa characteristics include the progressive motility, viability and morphological defect rates were evaluated in various times of semen storage period. The effects of vitamin C, zinc and storage periods of semen on spermatozoa progressive motility, viability and morphological defect rates were significant $(\mathrm{P}<0.05)$. Also, comparison the means by Duncan test revealed that the highest motility and viability rates and lowest morphological defect rates were observed in zero levels of vitamin $\mathrm{C}$ and zinc $(\mathrm{P}<0.05)$. Therefore, vitamin $\mathrm{C}$ and zinc have no improved effects on motility, viability and morphological defect rates of spermatozoa in liquid storage condition of Arabian ram.
\end{abstract}

Keywords: Arabi ram, Semen quality, Vitamin C, Zinc 